\title{
Patogenicidade de isolados de Beauveria bassiana para ovos, larvas e ninfas ingurgitadas de Rhipicephalus sanguineus
}

\author{
Pathogenicity of Beauveria bassiana isolates towards eggs, larvae and engorged \\ nymphs of Rhipicephalus sanguineus
}

\author{
Nancy Prette ${ }^{1}$ Antonio Carlos Monteiro ${ }^{2}$ Marcos Valério Garcia ${ }^{3}$ \\ Vando Edesio Soares ${ }^{4}$
}

\section{RESUMO}

O presente trabalho objetivou avaliar a ação dos isolados JAB 07, CB 7 e AM 9 do fungo Beauveria bassiana para ovos, larvas e ninfas ingurgitadas de Rhipicephalus sanguineus. Padronizou-se a concentração de $10^{9}$ conídios $m L^{-1}$ para cada isolado e por meio de diluições seriadas, obtiveram-se as suspensões com concentrações de $10^{8}$ e $10^{7} \mathrm{con}$. $m L^{-1}$. Preparou-se um bioensaio para cada fase do ciclo de vida do carrapato. Cada ensaio foi composto por 10 grupos tratamentos, formados por um isolado do fungo e uma concentração de conídios, e um grupo controle contendo apenas o veículo das suspensões. Os isolados do fungo, aplicados nas diferentes concentrações de esporos, causaram redução no percentual de eclosão de larvas oriundas de ovos infectados $(0,7$ a $12,1 \%$ de eclosão) e no percentual de ecdise de larvas (4,7 a 33,7\% de ecdise) e ninfas (0 a 16,7\% de ecdise). Não houve diferença entre os isolados $(P>0,05)$ quanto à infecção de ovos, mas a aplicação de $10^{9}$ con. $/ \mathrm{mL}$ de JAB 07 e AM 9 promoveu redução significativa da eclosão de larvas, em relação à concentração de $10^{7} \mathrm{con}$. $\mathrm{mL}^{-1}$. O isolado $C B 7$ foi o mais eficaz na redução do número de ecdises de larvas. Os isolados e as concentrações de conídios não diferiram $(P>0,05)$ quanto à capacidade de redução do percentual de ecdise de ninfas, mas evidenciou-se intensa atividade patogênica do fungo.

Palavras-chave: controle biológico, controle microbiano, fungo entomopatogênico, carrapato.

\begin{abstract}
The aim of the present study was to determine the action of isolates JAB 07, CB 7 and AM 9 of the fungus Beauveria bassiana on eggs, larvae and engorged nymphs of Rhipicephalus sanguineus tick. A concentration of $10^{9}$ conidia $\mathrm{ml}^{-1}$ was standardized for each isolate and suspensions containing $10^{8}$ and $10^{7}$ conidia $\mathrm{ml}^{-1}$ were obtained by serial dilution. A bioassay was prepared for each phase of the life cycle of the tick. Each assay comprised 10 treatment groups, consisting of one fungal isolate and one conidial concentration, and one control group containing only the vehicle of suspensions. For the eggs infection $0.1 \mathrm{~g}$ (about 270 eggs) were placed at $1 \mathrm{ml}$ of suspension at different concentrations, each engorged larvae group $(n=250)$ and engorged nymphs $(n=50)$ received $2 \mathrm{ml}$ of one conidial suspension. Thereafter, eggs, larvae and nymphs were placed in on incubator at $27 \pm 1{ }^{\circ} \mathrm{C}$ and at a humidity above $80 \%$. The fungal isolates applied at different spore concentrations caused a reduction in percent hatching of larvae derived from infected eggs ( 0.7 to $12.1 \%$ of hatching) and in percent ecdysis of larvae (4.7 to $33.7 \%$ of ecdysis) and nymphs (0 to $16.7 \%$ of ecdysis). No difference in the infection of eggs was observed between isolates $(P>0.05)$, but the application of $10^{9}$ conidia/ml of JAB 07 and AM 9 significantly reduced the hatching of larvae compared to the concentrations of $10^{7}$ conidia $\mathrm{ml}^{-1}$. The isolate $C B 7$ was more efficient in reducing the number of larval ecdysis. The isolates and concentrations tested didn't differ $(P>0.05)$ in their ability to reduce percent ecdysis of nymphs, but an intense pathogenic activity of the fungus was observed.
\end{abstract}

'Programa de Pós-graduação em Microbiologia (PPGM), Faculdade de Ciências Agrárias e Veterinárias (FCAV), Universidade Estadual Paulista (UNESP), Jaboticabal, SP, Brasil. E-mail: nprette@ hotmail.com

${ }^{2}$ Departamento de Produção Vegetal, FCAV, UNESP. Via de Acesso Prof. Paulo Donato Castellane, s/n. 14844-900. Jaboticabal, SP, Brasil. E-mail: montecar@fcav.unesp.br

${ }^{3}$ PPGM, FCAV, UNESP, Jaboticabal, SP, Brasil. E-mail: marcos@ fcav.unesp.br

${ }^{4}$ Centro de Pesquisas em Sanidade Animal (CPPar), FCAV, UNESP, Jaboticabal, SP, Brasil. E-mail: soares@ fcav.unesp.br 
Key words: biological control, entomopathogenic fungus, microbial control, tick.

\section{INTRODUÇÃO}

A ocorrência de carrapatos no Brasil varia de acordo com o ambiente onde o hospedeiro vive (LABRUNA \& PEREIRA, 2001). O carrapato Rhipicephalus sanguineus (Latreille, 1806) é, provavelmente, a espécie de maior disseminação mundial na atualidade (PEGRAM, et al., 1987), pela ampla distribuição de seu hospedeiro natural que é o cão, e por possuir hábitos nidícolas. Originário da região Afrotropical, o ixodídeo foi introduzido no Brasil possivelmente a partir do século XVI, com a chegada dos colonizadores europeus e seus animais domésticos (LABRUNA \& PEREIRA, 2001).

É um ectoparasito que se caracteriza pela hematofagia em animais de sangue quente, preferindo o cão como principal hospedeiro, mas pode parasitar cavalos, bovinos e até mesmo o homem. GODDARD (1989) verificou que há relação entre o número crescente de casos registrados de erliquiose humana devido à infecção por Ehrlichia canis e o aumento de parasitismo humano por $\boldsymbol{R}$. sanguineus. O parasito pode ainda transmitir ao homem, outras espécies de riquétisias, como a Rickettsia rickettsii e a Rickettsia conorii, agentes casuais, respectivamente, da Febre Maculosa das Montanhas Rochosas no México e na América do Sul e da Febre Botonosa na região mediterrânea do sul da Europa e da África do Norte (SWANGO et al., 1992). O cão tem um importante papel na epidemiologia dessas doenças que ocorrem no homem, pois é ele que leva os carrapatos infectados para o ambiente humano (ACHA \& SZYFRES, 1986).

O controle das infestações de $\boldsymbol{R}$. sanguineus é realizado principalmente com a aplicação de produtos químicos sintéticos (acaricidas). Recentemente, MILLER et al. (2001) verificaram resistência do carrapato a alguns dos princípios ativos usados em formulações comerciais de acaricidas. Além disso, vários destes princípios ativos podem causar intoxicações, atingindo crianças, que geralmente são mais susceptíveis. Assim, a utilização exclusiva de carrapaticidas químicos é cada dia menos viável em termos práticos e econômicos, tornando-se necessária a adoção de métodos alternativos a serem empregados em sistemas integrados de controle (BARROS \& EVANS, 1989). Estes sistemas visam também atenuar os problemas causados pelo uso indiscriminado de carrapaticidas, que além de promover resistência a vários princípios ativos, causam poluição ambiental e intoxicação ao homem (PAIÃO et al., 2001).
O controle microbiano destaca-se como promissor, sendo bactérias e fungos os microrganismos mais estudados no controle do Rhipicephalus. De acordo com ALVES (1998), os fungos entomopatogênicos englobam cerca de 90 gêneros e 700 espécies, estando entre os bioagentes mais utilizados no controle biológico de pragas. $\mathrm{O}$ uso dos fungos é viabilizado pelo fato de não serem prejudiciais ao homem e outros animais homeotermos, pois dificilmente conseguem se desenvolver na faixa de temperatura dos diferentes mamíferos. Algumas espécies têm sido estudadas como patógenos de carrapatos, especialmente "in vitro", mas também "in vivo". Recentemente, GARCIA et al. (2004) descreveram os eventos envolvidos no mecanismo de adesão, penetração e colonização de fêmeas ingurgitadas de $\boldsymbol{R}$. sanguineus por M. anisopliae. Apesar dos avanços obtidos nos últimos anos, ainda são poucos os trabalhos que tratam do uso de fungos para o controle biológico de carrapatos, e em particular do $R$. sanguineus.

O objetivo do presente trabalho foi verificar a suscetibilidade de ovos, larvas e ninfas de $\boldsymbol{R}$. sanguineus à ação de três isolados do fungo $\boldsymbol{B}$. bassiana, inoculados em diferentes concentrações de esporos "in vitro", procurando determinar os isolados mais patogênicos e as concentrações mais efetivas para o controle das fases não parasitarias do carrapato.

\section{MATERIAL E MÉTODOS}

A colônia do carrapato $\boldsymbol{R}$. sanguineus foi mantida junto ao Laboratório de Imunopatologia do Departamento de Patologia Veterinária da Faculdade de Ciências Agrárias e Veterinárias (FCAV) da Universidade Estadual Paulista (Unesp).

Utilizaram-se os isolados JAB 07, CB 7 e AM 9 (obtidos, respectivamente, de Musca domestica, Anthonomus grandi e Deois incompleta) do fungo Beauveria bassiana (Bals.) Vuill., mantidos em culturas estoques na coleção do Laboratório de Microbiologia do Departamento de Produção Vegetal da FCAVUnesp.

Os isolados foram cultivados espalhandose uma suspensão de conídios sobre o meio BDA [200 $\mathrm{g}$ de batata (B), 20g de dextrose (D) e 20g de ágar (A)] contido em placas de Petri, e incubando-se em estufa a $27 \pm 1^{\circ} \mathrm{C}$, durante 20 dias, com ausência de iluminação. A viabilidade dos isolados, assim cultivados, foi determinada pelo método de contagem de conídios germinados em lâmina de microscopia ao microscópio ótico (PAIÃO, 2000), obtendo-se os seguintes valores: CB 7: 96,6\%, JAB 07: 95,3\%, AM 9: 97,5\%. 
As suspensões de conídios foram preparadas a partir de colônias jovens dos isolados. Conídios da superfície das colônias foram transferidos, por meio de uma alça de níquel-cromo flambada e resfriada, para tubos de ensaio contendo $20 \mathrm{~mL}$ de solução de Tween $80^{\circledR}$ a $0,1 \%$ (v/v). A quantificação dos conídios presentes foi feita em câmara de Neubauer com amostras devidamente homogeneizadas de cada suspensão fúngica. Padronizou-se a concentração de $10^{9}$ con. $\mathrm{mL}^{-1}$ para cada isolado e por meio de diluições em série, realizadas em tubos contendo solução salina de $\mathrm{NaCl}$ a $0,89 \%(\mathrm{p} / \mathrm{v})$, obtiveram-se as concentrações de $10^{8}$ e $10^{7}$ con. $\mathrm{mL}^{-1}$.

Prepararam-se três bioensaios, um para cada fase do ciclo biológico do carrapato, ovos, larvas e ninfas. Cada bioensaio foi composto por 10 grupos (tratamentos), sendo cada um formado por um isolado do fungo e uma concentração de conídios, e um grupo controle contendo apenas o veículo das suspensões, buscando sempre trabalhar grupos homogêneos.

Utilizaram-se ovos de $\boldsymbol{R}$. sanguineus postos entre o primeiro e o sétimo dia de postura, visando diminuir possíveis efeitos causados pela diferença nos estádios de desenvolvimento dos ovos. A postura foi removida e separada em grupos de $0,10 \mathrm{mg}$, (cerca de 270 ovos) com três repetições para cada tratamento. Os ovos foram colocados em seringas de plástico adaptadas pela remoção da extremidade de encaixe da agulha, recebendo $1 \mathrm{~mL}$ de uma das suspensões de conídios que representou um dos tratamentos do bioensaio.

Após um minuto de agitação manual, o excesso de líquido foi retirado com papel de filtro. As seringas foram identificadas, vedadas com algodão e mantidas em estufa a $27 \pm 1^{\circ} \mathrm{C}$ e umidade relativa do ar acima de $80 \%$. Decorridos 20 dias foi determinada a percentagem de eclosão através de amostragem, transferindo o conteúdo de cada seringa para um bequer contendo $50 \mathrm{~mL}$ de água e etanol $(80: 20 \mathrm{v} / \mathrm{v})$. Com a solução em agitação manual, foi retirado com pipeta $10 \%$ do volume, colocando-o em uma placa de Petri para contagem dos ovos remanescentes, utilizando-se microscópio estereoscópico (PAIÃO et al., 2001). As contagens foram individualizadas, ou seja, para cada repetição em cada tratamento, fez-se uma contagem.

Larvas e ninfas provenientes da colônia foram colocadas em coelhos para ingurgitarem, em câmaras de plástico transparente coladas no dorso do animal com material atóxico (Britania Adhesives P4104 Latex, Inglaterra) (BECHARA et al., 1994).
As larvas e ninfas foram tratadas logo após o seu despreendimento do animal nas câmaras de alimentação. Grupos de 250 larvas e de 50 ninfas, ambas ingurgitadas, foram banhados em $2 \mathrm{~mL}$ de uma das suspensões de conídios que representou um dos tratamentos do ensaio, contidas em copos descartáveis de plástico. Após agitação manual por um minuto, retirou-se o excesso de líquido com papel de filtro e mantiveram-se os grupos individualizados em frascos etiquetados e acondicionados em estufa a $27 \pm 1^{\circ} \mathrm{C}$ e umidade relativa do ar acima de $80 \%$. Os grupos controle foram banhados em solução de Tween $80^{\circledR} \mathrm{e}$ mantidos nas mesmas condições dos grupos tratados. A avaliação da ecdise dos grupos tratados foi realizada após completa ecdise do grupo controle, por meio de contagem manual de cada grupo, em microscópio estereoscópico.

Os experimentos foram realizados num esquema fatorial com dois fatores $\mathrm{x}$ testemunha, sendo um fator o isolado e o outro a concentração de conídios da suspensão (três isolados e três concentrações). Para cada tratamento, fizeram-se três repetições. Os resultados foram submetidos à análise de variância pelo teste $\mathrm{F}$ e as médias dos tratamentos foram comparadas pelo teste de Tukey a 5\% de probabilidade de erro. Os dados expressos em porcentagens foram transformados, para a realização das análises, em arco seno da raiz de $\mathrm{x}(\mathrm{x} / 100)^{1 / 2}$. Efetuou-se a análise de regressão para as concentrações de esporos para cada isolado.

\section{RESULTADOS E DISCUSSÃO}

O percentual de eclosão de larvas, a partir dos ovos inoculados, foi significativamente influenciado pelos isolados e pelas concentrações de conídios, conforme se verifica na Tabela 1 pelo confronto dos grupos tratados com o grupo controle. Entre os isolados, não ocorreram diferenças significativas, mas o mesmo não foi observado para as concentrações de conídios, visto que para JAB 07 e AM 9 a concentração de $10^{9} \mathrm{con}$. $\mathrm{mL}^{-1}$ diferiu da concentração $10^{7}$ con. $\mathrm{mL}^{-1}$.

O percentual de eclosão variou no grupo testemunha de 95,0 a 98,3\% e nos grupos tratados de 0,7 a $12,1 \%$. Na comparação múltipla de médias dos percentuais da eclosão de larvas, os grupos tratados diferiram significativamente do grupo controle $(\mathrm{P}<0,05)$.

Na figura 1, pode-se constatar maior eficácia dos isolados, quando estes foram inoculados em suspensões com concentrações de $10^{9} \mathrm{con}$. $\mathrm{mL}^{-1}$; 
Tabela 1 - Eclosão e ecdise de larvas e ninfas ingurgitadas de Rhipicephalus sanguineus após infecção com os isolados JAB 07, CB 7 e AM 9 de Beauveria bassiana, aplicados em diferentes concentrações de conídios.

\begin{tabular}{|c|c|c|c|c|c|c|}
\hline \multirow{2}{*}{ Isolados } & \multicolumn{2}{|c|}{ Ovos } & \multicolumn{2}{|c|}{ Larvas } & \multicolumn{2}{|c|}{ Ninfas } \\
\hline & Conc. (con./mL) & Eclosão de larvas(\%) & Conc. (con./mL) & Ecdise $(\%)$ & Conc. (con./mL) & Ecdise $(\%)$ \\
\hline \multirow{3}{*}{ JAB 07} & $1,36 \times 10^{7}$ & $11,98 \mathrm{aB}$ & $1,60 \times 10^{7}$ & $33,73 \mathrm{aB}$ & $1,60 \times 10^{7}$ & $2,00 \mathrm{aB}$ \\
\hline & $1,36 \times 10^{8}$ & 4,69abB & $1,60 \times 10^{8}$ & $23,07 \mathrm{aB}$ & $1,60 \times 10^{8}$ & $10,00 \mathrm{aB}$ \\
\hline & $1,36 \times 10^{9}$ & $1,36 \mathrm{bB}$ & $1,60 \times 10^{9}$ & $13,87 \mathrm{aB}$ & $1,60 \times 10^{9}$ & $4,67 \mathrm{aB}$ \\
\hline \multirow{3}{*}{ CB 7} & $1,30 \times 10^{7}$ & $8,64 \mathrm{aB}$ & $1,62 \times 10^{7}$ & $16,67 \mathrm{aB}$ & $1,62 \times 10^{7}$ & $2,00 \mathrm{aB}$ \\
\hline & $1,30 \times 10^{8}$ & $3,95 \mathrm{aB}$ & $1,62 \times 10^{8}$ & $4,67 \mathrm{aB}$ & $1,62 \times 10^{8}$ & $16,67 \mathrm{aB}$ \\
\hline & $1,30 \times 10^{9}$ & $4,44 \mathrm{aB}$ & $1,62 \times 10^{9}$ & $19,60 \mathrm{aB}$ & $1,62 \times 10^{9}$ & $2,67 \mathrm{aB}$ \\
\hline \multirow{3}{*}{ AM 9} & $1,48 \times 10^{7}$ & $12,10 \mathrm{aB}$ & $1,60 \times 10^{7}$ & $21,73 \mathrm{aB}$ & $1,60 \times 10^{7}$ & $13,33 \mathrm{aB}$ \\
\hline & $1,48 \times 10^{8}$ & 7,04abB & $1,60 \times 10^{8}$ & $26,67 \mathrm{aB}$ & $1,60 \times 10^{8}$ & $2,00 \mathrm{aB}$ \\
\hline & $1,48 \times 10^{9}$ & $0,74 \mathrm{bB}$ & $1,60 \times 10^{9}$ & $10,27 \mathrm{aB}$ & $1,60 \times 10^{9}$ & $0,00 \mathrm{aB}$ \\
\hline \multirow{3}{*}{ Controle } & $\mathrm{R} 1$ & $98,33 \mathrm{aA}$ & $\mathrm{R} 1$ & $94,13 \mathrm{aA}$ & $\mathrm{R} 1$ & $100,00 \mathrm{aA}$ \\
\hline & $\mathrm{R} 2$ & $96,67 \mathrm{aA}$ & $\mathrm{R} 2$ & $96,26 \mathrm{aA}$ & $\mathrm{R} 2$ & $93,33 \mathrm{aA}$ \\
\hline & R3 & $95,00 \mathrm{aA}$ & R3 & $93,47 \mathrm{aA}$ & R3 & $100,00 \mathrm{aA}$ \\
\hline Causa da variação & GL & $\mathrm{F}$ & GL & $\mathrm{F}$ & GL & $\mathrm{F}$ \\
\hline Controle vs Fatorial & 1 & $1126,84 * *$ & 1 & $310,55 * *$ & 1 & $329,12 * *$ \\
\hline Controle & 2 & $0,99 \mathrm{NS}$ & 2 & $0,18 \mathrm{NS}$ & 2 & $0,65 \mathrm{NS}$ \\
\hline Isolados (I) & 2 & $1,73 \mathrm{NS}$ & 2 & $3,86 *$ & 2 & $0,02 \mathrm{NS}$ \\
\hline Concentrações (C) & 2 & $14,30 * *$ & 2 & $2,35 \mathrm{NS}$ & 2 & $1,84 \mathrm{NS}$ \\
\hline (I) vs (C) & 4 & $1,49 \mathrm{NS}$ & 4 & $1,94 \mathrm{NS}$ & 4 & $1,22 \mathrm{NS}$ \\
\hline Tratamentos & 11 & $106,08 * *$ & 11 & $30,10 * *$ & 11 & $30,82 * *$ \\
\hline Resíduo & 24 & - & 24 & - & 24 & - \\
\hline Total & 35 & - & 35 & - & 35 & - \\
\hline
\end{tabular}

NS: não significativo. *Significativo a $5 \%$ de probabilidade. ** Significativo a $1 \%$ de probabilidade.

$\mathrm{R} 1, \mathrm{R} 2, \mathrm{R} 3$ : repetições 1,2 , e 3, respectivamente.

Médias de concentrações dentro de cada isolado seguidas de mesma letra minúscula na coluna, não diferem entre si (P>0,05).

Médias de isolados dentro de cada concentração seguidas de mesma letra maiúscula na coluna, não diferem entre si (P>0,05).

Coeficiente de variação de ovos: $18,85 \%$. Coeficiente de variação de larvas: $20,70 \%$. Coeficiente de variação de ninfas: $37,49 \%$.

observou-se uma relação inversamente proporcional entre a concentração de esporos e o percentual de eclosão.

Dados semelhantes a estes foram obtidos por MONTEIRO et al. (1998) que observaram acentuada redução no percentual de eclosão de larvas de R. sanguineus, promovidos por Boophilus bassiana e $M$. anisopliae usados na concentração $10^{8}$ con./mL. BITTENCOURT et al. (1994) e PAIÃO et al. (2001) estudando, respectivamente, a ação de M. anisopliae e B. bassiana sobre ovos de Boophilus microplus, obtiveram os melhores resultados com os tratamentos contendo as maiores concentrações de esporos.

Entretanto, FERNANDES et al. (2001) não encontraram diferença estatística entre o grupo controle e o percentual de eclosão de larvas de $\boldsymbol{B}$. microplus oriundas de ovos tratados com diferentes isolados de $\boldsymbol{B}$. bassiana.
Pela análise dos dados de ecdise de larvas de $\boldsymbol{R}$. sanguineus infectadas com os três isolados de B. bassiana (Tabela 1), verificou-se que houve diferença significativa entre os isolados JAB 07 e CB 7 , mas não entre as concentrações de conídios. $\mathrm{O}$ isolado CB 7 foi o mais eficaz na redução do número de ecdises de larvas. O percentual de ecdise observado no grupo testemunha diferiu significativamente dos grupos tratados, quando aplicada a diferença mínima significativa (DMS) às médias dos tratamentos.

Assim como para os ovos infectados, pode-se constatar uma tendência de redução de ecdise de larvas, à medida que se aumentou a concentração de esporos nas suspensões inoculadas nas larvas (Figura 2). BITTENCOURT et al. (1995) verificaram que houve aumento na mortalidade de larvas de B. microplus devido à ação do fungo $\boldsymbol{B}$. bassiana e PAIÃO et al. (2001) observaram que, na maioria dos tratamentos analisados, o aumento da mortalidade de larvas foi 


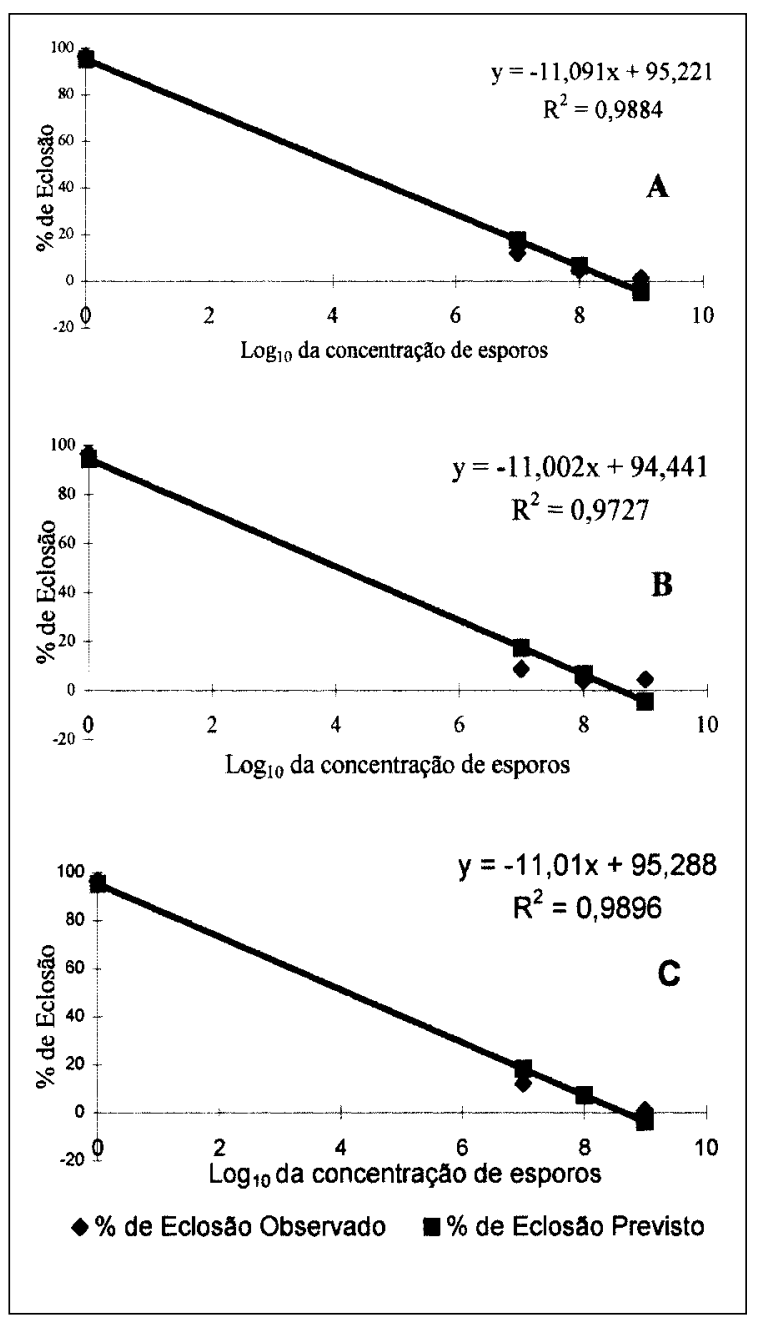

Figura 1 - Resultados da análise de regresão dos dados de eclosão das larvas de $\boldsymbol{R}$. sanguineos após infecção de ovos com os isolados JAB 07 (A), CB 7 (B) e AM 9 (C) de B.bassiana

proporcional ao aumento das concentrações de esporos.

De acordo com KAAYA \& HASSAN (2000), larvas não alimentadas de Rhipicephalus appendiculatus e Amblyomma variegatum, apresentaram $100 \%$ de mortalidade quando tratadas com $10^{9}$ con./mL de M. anisopliae e B. bassiana. Larvas ingurgitadas de $\boldsymbol{R}$. sanguineus banhadas com suspensão de esporos na concentração $1 \times 10^{7}$ con./ $\mathrm{mL}$, dos fungos B. bassiana, Paecilomyces fumosoreus e Metarhizium flavoviride apresentam mortalidade de 10 a $20 \%$ e quando tratadas com a mesma concentração com o fungo M. anisopliae, observou-se mortalidade de 49,1 a 82,6\% (SAMISH et al., 2001).
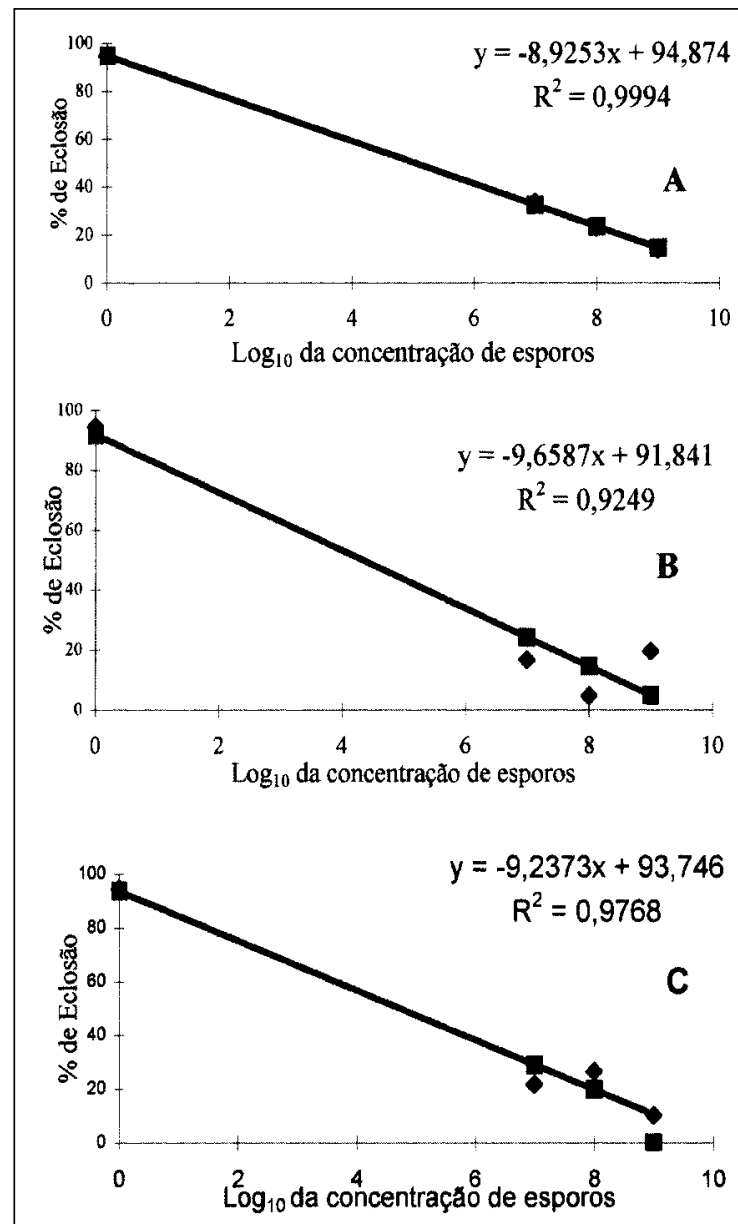

-\% de ecdise observada $\quad \square \%$ de ecdise prevista

Figura 2 - Resultados da análise de regresão dos dados de ecdise das larvas de $\boldsymbol{R}$. sanguineos após infecção com os isolados JAB 07 (A), CB 7 (B) e AM 9 (C) de B.bassiana

O percentual de ecdise de larvas ingurgitadas de $\boldsymbol{R}$. sanguineus do grupo controle variou de 93,5 a $96,3 \%$ e, nos grupos tratados, de 4,7 a $33,7 \%$. Os dados apresentados mostraram a patogenicidade dos três isolados de B. bassiana para o estádio larval ingurgitado do carrapato $\boldsymbol{R}$. sanguineus.

Analisando os dados da ação do fungo sobre ninfas, verificamos que não ocorreu diferença significativa entre os isolados e entre as concentrações quanto à capacidade de redução no percentual de ecdises (Tabela 1). Quando aplicada a DMS às médias dos tratamentos, os grupos tratados diferiram do grupo testemunha.

Os resultados obtidos com ninfas ingurgitadas demostraram acentuada redução no

Ciência Rural, v.35, n.4, jul-ago, 2005. 
percentual de ecdise que variou de 0 a $16,7 \%$ nos grupos tratados, enquanto no controle obteve-se de 93,3 a $100 \%$. Novamente, como já observado nos ensaios anteriores, verificou-se uma tendência de redução de ecdises de ninfas à medida que aumentou a concentração de conídios das suspensões (Figura 3).

Estes resultados são similares aos obtidos por outros pesquisadores. Estudando a patogenicidade dos fungos B. bassiana e M. anisopliae para o carrapato Argas persicargas persicus, SEWIFY \& HABIB (2001) encontraram que o primeiro ínstar ninfal teve alta suscetibilidade para ambos os fungos e que as fêmeas não ingurgitadas foram mais susceptíveis do que as fêmeas ingurgitadas, sugerindo, segundo os autores, que estes fungos tenham bom potencial para o controle de carrapato de ave.

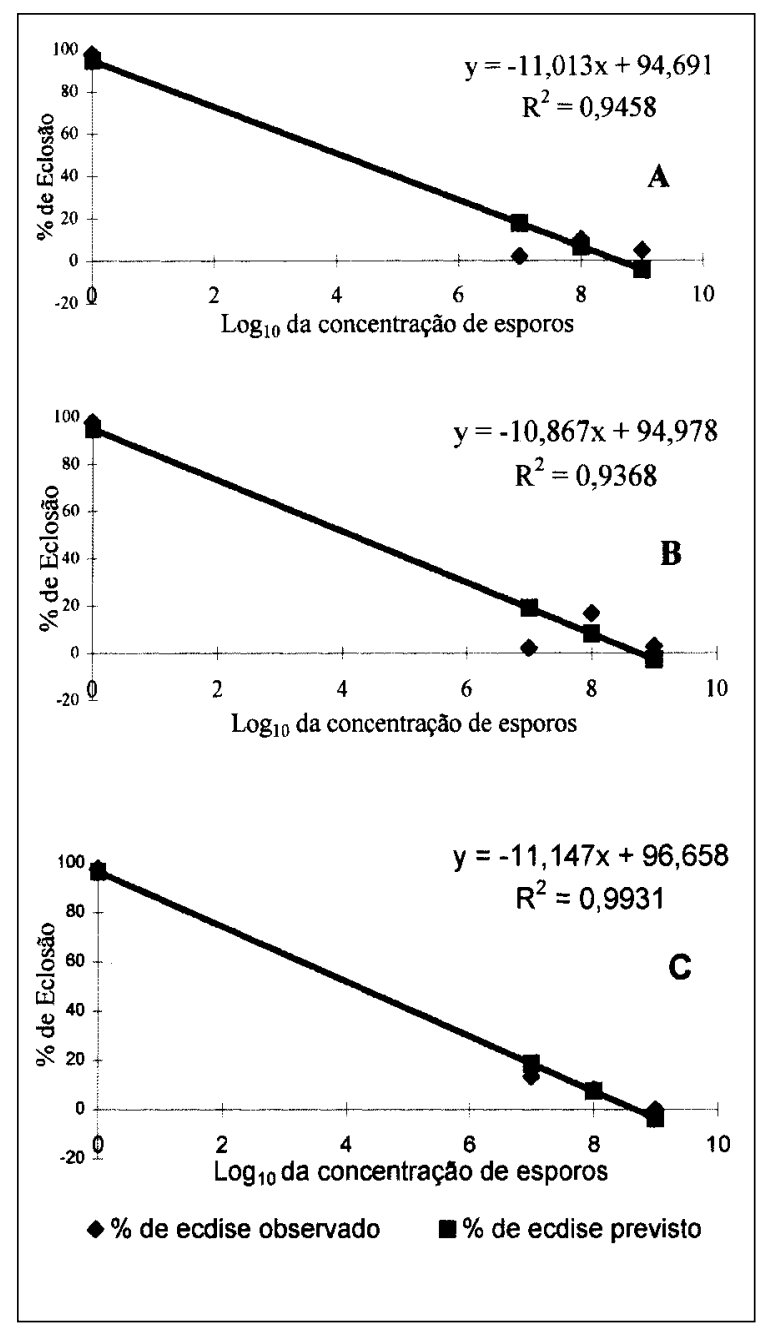

Figura 3 - Resultados da análise de regresão dos dados de ecdise de ninfas de $\boldsymbol{R}$. sanguineos após infecção com os isolados JAB 07 (A), CB 7 (B) e AM 9 (C) de B.bassiana
Analisando o efeito de dois isolados de $\boldsymbol{B}$. bassiana, na sobrevivência de ninfas não alimentadas de $\boldsymbol{R}$. sanguineus, BARBOSA et al. (1997) observaram que a mortalidade nos grupos tratados com suspensões de $10^{6}$ e $10^{8}$ con./mL foi de $100 \%$. KAAYA \& HASSAN (2000) encontraram mortalidade de 80 a $100 \%$ em ninfas não alimentadas de Rhipicephalus appendiculatus e Amblyoma variegatum, quando tratadas com $10^{9}$ con. $/ \mathrm{mL}$ de $\boldsymbol{M}$. anisopliae e $\boldsymbol{B}$. bassiana.

Ao aplicarem o fungo $M$. anisopliae associado a um gel e concentrado emulsionável sobre ninfas ingurgitadas de R. sanguineus, REIS et al. (2003) verificaram redução de $10 \%$ na sobrevivência de ninfas. SAMISH et al. (2001) avaliaram a patogenicidade de alguns fungos para ninfas ingurgitadas e ninfas não alimentadas de $\boldsymbol{R}$. sanguineus, observando que ambas foram mais susceptíveis a $\boldsymbol{M}$. anisopliae e $\boldsymbol{M}$. flavorividae.

\section{CONCLUSÃO}

Os isolados JAB 07, CB 7 e AM 9 do fungo Beauveria bassiana são patogênicos para ovos, larvas e ninfas ingurgitadas de Rhipicephalus sanguineus, havendo maior eficiência da ação patogênica à medida que aumenta a concentração de esporos da suspensão usada para infectar os diferentes estágios do ciclo de vida do carrapato.

\section{AGRADECIMENTOS}

Os autores agradecem à Fundação de Amparo à Pesquisa do Estado de São Paulo (FAPESP: 01/12558-9), pela concessão da bolsa de mestrado à primeira autora.

\section{REFERÊNCIAS}

ACHA, P.N.; SZYFRES, B. Zoonosis y enfermedades transmisibles comunes al hombre y los animales. 2.ed. Washington : OPS/OMS, 1986. 989p.

ALVES, S.B. Controle microbiano de insetos. 2.ed. São Paulo : FEALQ, 1998. 1163p.

BARBOSA, J.V. et al. Efeitos de dois isolados do fungo Beauveria bassiana (Bals.) Vuill, sobre a muda larval e sobrevivência de ninfas de Rhipicephalus sanguineus (Latreille, 1806) (Acari: Ixodidae). Revista Brasileira de Parasitologia Veterinária, Rio de Janeiro, v.6, n.1, p.5356, 1997.

BARROS, T.A.M; EVANS, D.E. Ação de gramíneas forrageiras em larvas infestantes do carrapato de bovinos, Boophilus microplus. Pesquisa Veterinária Brasileira, Rio de Janeiro, v.9, n.1/2, p.17-21, 1989. 
BECHARA, G.H. et al. Immunisation of dogs, hamsters and guinea pigs against Rhipicephalus sanguineus using crude unfed adult tick extracts. Veterinary Parasitology, Amsterdam, v.52, p.79-90, 1994.

BITTENCOURT, V.R.E.P. et al. Ação do fungo Metarhizium anisopliae em ovos e larvas do carrapato Boophilus microplus. Revista da Universidade Rural, Série Ciência da Vida, Rio de Janeiro, v.16, n.1-2, p.49-55, 1994.

BITTENCOURT, V.R.E.P. et al. Eficácia in vitro dos isolados 747 e 986 do fungo Beauveria bassiana no carrapato Boophilus microplus. Revista Brasileira de Parasitologia Veterinária, Rio de Janeiro, v.4, supl.1.1, p.86, 1995.

FERNANDES, É.K.K. et al. Patogenicidade de diferentes linhagens de Beauveria bassiana (Bals) Vuill. em ovos de Boophilus microplus in vitro. In: SIMPOSIO DE CONTROLE BIOLÓGICO, 7., 2001, Poços de Caldas, MG. Resumos... Poços de Caldas : Universidade Federal de Lavras e Embrapa (CNPMS), 2001. V.1. 472p. p.353.

GARCIA, M.V. et al. Colonização e lesão em fêmeas ingurgitadas do carrapato Rhipicephalus sanguineus causadas pelo fungo Metarhizium anisopliae. Ciência Rural, Santa Maria, v.34, n.5, p.1513-1518, 2004

GODDARD, J. Focus of human parasitism by the brown dog tick, Rhipicephalus sanguineus (Acari: Ixodidae). Journal of Medical Entomology, Lanham, v.26, n.6, p.628-629, 1989

KAAYA, G.P.; HASSAN, S. Entomogenous fungi as promising biopesticides for tick control. Experimental and Applied Acarology, Amsterdam, v.24, p.913-926, 2000.

LABRUNA, M.B.; PEREIRA, M.C. Carrapato em cães. Clinica Veterinária, São Paulo, v.6, p.24-32, 2001.

MILLER, R.J. et al. Characterization of acaricide resistance in Rhipicephalus sanguineus (Latreille) (Acari: Ixodidae) collected from the Corozal Army Veterinary Quarentine Center, Panama. Journal of Medical Entomology, Lanham, v.38, n.2, p.298-302, 2001.
MONTEIRO, S.G. et al. Ação dos fungos Beauveria bassiana e Metarhizium anisopliae em larvas do carrapato Rhipicephalus sanguineus. Ciência Rural, Santa Maria, v.28, n.3, p.461-466, 1998.

PAIÃO, J.C.V. Compatibilidade dos fungos Beauveria bassiana e Metarhizium anisopliae com carrapaticidas químicos utilizados no controle de Boophilus microplus (Acari: Ixodidae). 2000. 55f. Dissertação (Mestrado em Microbiologia) - Programa de Pós-graduação em Microbiologia. Faculdade de Ciências Agrárias e Veterinárias, Unesp.

PAIÃO, J.C.V. et al. Susceptibility of the cattle tick Boophilus microplus (Acari: Ixodidae) to isolates of the fungus Beauveria bassiana. World Journal of Microbiology \& Biotechnology, Dordrecht, v.17, n.3, p.245-251, 2001.

PEGRAM, R.G. et al. Clarification of the Rhipicephalus sanguineus group (Acari, Ixodoidea, Ixodidae). II. $\boldsymbol{R}$. sanguineus (Latreille, 1806) and related species. Systematic Parasitology, Dordrecht, v.10, p.27-44, 1987.

REIS, R.C.S. et al. Patogenicidade de formulações fúngicas na viabilidade de ninfas alimentadas do carrapato Rhipicephalus sanguineus (Latreille, 1806) (Acari: Ixodidae). In: SIMPOSIO DE CONTROLE BIOLÓGICO, 8., 2003, São Pedro, SP. Resumos... São Pedro : Sociedade Entomológica do Brasil, 2001. V.1. 206p. p.96.

SAMISH, M. et al. Pathogenicity of entomopathogenic fungi to different developmental stages of Rhipicephalus sanguineus (Acari: Ixodidae). Journal of Parasitology, Lawrence, v.87, n.6, p.1355-1359, 2001.

SEWIFY, G.H.; HABIB, S.M. Biological control of the tick fowl Argas persicargas by the entomopathogenic fungi Beauveria bassiana and Metarhizium anisopliae. Anzeigerfuer- Schaedlingskunde, Hamburg, v.74, n.5, p.121-123, 2001.

SWANGO, L.J. et al. Infecções bacterianas, riquetsiais, protozoais e outras. In: ETTINGER, S.J. Tratado de medicina veterinária interna: moléstias do cão e do gato. 3.ed. São Paulo : Manole, 1992. Cap.46, sec.3, p.277-311. 